\title{
Accurate Electrothermal Modeling of Thermoelectric Generators
}

\author{
Mohammad Javad Dousti ${ }^{1}$, Antonio Petraglia ${ }^{2}$, and Massoud Pedram ${ }^{1}$ \\ ${ }^{1}$ University of Southern California, USA and ${ }^{2}$ Federal University of Rio de Janeiro, Brazil \\ ${ }^{1}\{$ dousti,pedram $\} @$ usc.edu and ${ }^{2}$ antonio@pads.ufrj.br
}

\begin{abstract}
Thermoelectric generators (TEGs) provide a unique way for harvesting thermal energy. These devices are compact, durable, inexpensive, and scalable. Unfortunately, the conversion efficiency of TEGs is low. This requires careful design of energy harvesting systems including the interface circuitry between the TEG module and the load, with the purpose of minimizing power losses. In this paper, it is analytically shown that the traditional approach for estimating the internal resistance of TEGs may result in a significant loss of harvested power. This drawback comes from ignoring the dependence of the electrical behavior of TEGs on their thermal behavior. Accordingly, a systematic method for accurately determining the TEG input resistance is presented. Next, through a case study on automotive TEGs, it is shown that compared to prior art, more than $11 \%$ of power losses in the interface circuitry that lies between the TEG and the electrical load can be saved by the proposed modeling technique. In addition, it is demonstrated that the traditional approach would have resulted in a deviation from the target regulated voltage by as much as $59 \%$.
\end{abstract}

\section{INTRODUCTION}

Energy harvesting has gained significant attention due to the ever increasing demand for energy. Harvested energies are usually renewable energies (such as solar, wind, etc.) or otherwise wasted energies (like heat) [1]. Abundance and availability at no cost make harvesting of the electrical energy out of those sources quite attractive. One of such energy sources is heat, which can be converted into electricity by means of thermoelectric generators (TEGs). TEGs work based on the Seebeck effect, which converts a temperature gradient into a voltage.

TEGs have unique capabilities, which have made them a preferable choice compared to conventional energy sources (such as batteries) and other energy harvesting methods (such as solar cells). TEGs are: 1) Silent: TEGs have no moving part and are made of semiconductor materials and hence, generate no noise [2]. 2) Very durable: TEGs are reported to work for up to 30 years [2], which makes them ideal for remote or difficult-toreach locations and the outer space. For space missions beyond Mars, TEGs are the only means of energy harvesting, since the sunlight intensity drops significantly [3]. 3) Compact and lightweight: Each TEG can be manufactured to be as small as $0.5 \mathrm{~mm} \times 0.5 \mathrm{~mm} \times 100 \mu \mathrm{m}$ [4]. 4) Inexpensive: The cost of deploying TEGs compared to large generators or batteries (considering the replacement cost) is quite low [5]. 5) Scalable: TEG modules can be simply connected together to increase the amount of harvested energy [2].

Despite the aforesaid appealing characteristics, TEGs suffer from low conversion efficiency, which is imposed by two main factors. First, the Carnot cycle efficiency, which sets a theoretical upper bound on the conversion efficiency of thermal energy to work, can be quite low. Specifically, this efficiency is defined as $\eta_{\text {Carnot }}=\Delta T / T_{h}$, where $T_{h}$ is the temperature of the hot side and $\Delta T$ is the temperature difference between hot and cold sides. Clearly, when $\Delta T$ is small, the conversion efficiency is quite low. For instance, $30 \mathrm{~K}$ temperature difference in the room temperature $(300 \mathrm{~K})$ can provide up to $10 \%$ efficiency. The second limiting factor is the efficiency of the thermoelectric effect. The overall TEG efficiency can be formulated as [2]

$$
\eta_{T E G}=\frac{\Delta T}{T_{h}} \cdot \frac{\sqrt{1+Z T_{a v g}}-1}{\sqrt{1+Z T_{a v g}}+T_{c} / T_{h}},
$$

where $Z$ is the TEG figure of merit and $T_{a v g}=\left(T_{h}+T_{c}\right) / 2$. State-of-the-art TEGs have $Z T_{\text {avg }}$ value of 2.1 for $T_{a v g}=300 \mathrm{~K}$ $\left(27^{\circ} \mathrm{C}\right)$ [4]. For the same temperature difference used above, the efficiency of this TEG is equal to only $2.8 \%$, which is $72 \%$ lower than that of an ideal Carnot cycle. Evidently, the efficiency of TEGs is quite low. Low efficiency limits the usage of TEGs to low-power applications. Note that usually the overall energy of the source is rather low. For instance, you may consider the heat generated from the human body. This factor also limits the amount of harvested energy. Devices with power consumption of $100 \mathrm{~mW}$ or less are ideal targets to be powered by thermoelectric generators, whereas devices with higher power consumption require larger temperature gradient in order to be powered by TEGs.

The process of converting the temperature difference to usable electrical energy involves two steps. First, TEGs convert the temperature difference into an electrical voltage which is usually not suitable for the load and needs to be regulated. Next, this voltage is converted by an interface circuit to a regulated voltage required by the load or the energy storage element. This process is shown in Fig. 1. Note that in order to extract the maximum power from the generator and transfer it to the load, the interface circuit input resistance $\left(R_{\text {iface }}^{i n}\right)$ must be matched to the TEG internal resistance $\left(R_{T E G, N}^{i n}\right)$. This step is necessary to avoid losses in an already-low harvested energy.

In the prior art (such as [1], [3], [6], [7]), $R_{T E G}^{\text {in }}$ was set to be equal to the electrical resistance of the thermoelectric module $\left(R_{T E G}\right)$. In this paper, we first develop an electro-thermal model of TEGs. Using this model, an analytical methodology for determining $R_{T E G}^{i n}$ is presented. Next, we show that the electrical resistance of a TEG can differ from its internal resistance by more than $50 \%$. This difference comes from ignoring the dependence of the electrical behavior of TEG on its thermal behavior. Through a case study on automotive TEGs, we show that compared to prior art, more than $11 \%$ of power losses in the interface circuitry that lies between the TEG and the electrical load can be saved by the proposed modeling technique. In addition, Also, it is demonstrated that the load mismatch can cause the planned output voltage across the load to deviate as much as $59 \%$.

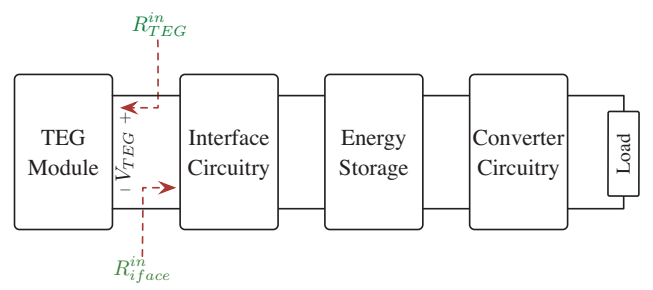

Fig. 1. High-level structure of a TEG harvesting system 
The remainder of this paper is organized as follows. Section II describes prior work. Next, Section III presents an electro-thermal model for TEGs and derive a methodology for determining their input resistances. Section IV presents simulation results including a case study on automotive TEGs (ATEGs). Finally, Section V concludes the paper.

\section{Previous WORK}

Research on thermoelectric generators is mainly divided into two parts. First, the manufacturing and assembly techniques in order to maximize TEG's figure of merit. Second, designing the interface circuit for maximally transferring the generated power to the load. The focus of this paper is on the latter part.

Much work has been conducted on designing interface circuits. Even though the electro-thermal model of TEGs are constructed (e.g., [8]), the internal resistance of $N$ TEGs $\left(R_{T E G, N}^{i n}\right)$ is claimed to be equal to the electrical resistance of the thermoelectric material and its associated contacts $\left(R_{T E G, N}\right)$. This modeling neglects the thermal resistance of TEG contacts and its effects on $R_{T E G, N}^{i n}$. Here we enumerate a few examples that consider $R_{T E G, N}^{i n}$ to be equal to $R_{T E G, N}^{i n}$.

Books [1] and [3] explain the basic equations for the amount of power that can be extracted from TEGs. In order to maximize the extracted power, they claim that the load should be matched with electrical resistivity of a TEG module. Solbrekken et al. [6] also use the electrical resistance of TEGs and adopt it as a relation to determine the internal resistance of TEGs. Lu et al. [7] use the electrical resistivity of TEGs to make a Thevenin equivalent circuit. As we will explain later, since TEGs are nonlinear circuits, the Thevenin theorem does not apply to them.

\section{ANALyticAl Modeling OF TEG InPUT RESISTANCE}

In this section, first an electro-thermal model of a TEG module is described. Next, considering the contact thermal and electrical resistances, we use this model to derive an accurate methodology for determining $R_{T E G}^{i n}$.

\section{A. TEG Electro-Thermal Model}

Thermoelectric generators are compact devices, which are made of pairs of $\mathrm{N}$ - and P-type semiconductor pellets. Usually, these pellets are fabricated from properly doped Bismuth Telluride $\left(\mathrm{Bi}_{2} \mathrm{Te}_{3}\right)$. When a temperature difference is applied across these pellets, current flows through pellets due to the Seebeck effect. The direction of generated current in an N-type pellet is opposite of that of a P-type pellet. Hence, to improve the amount of harvested energy and increase the overall generated voltage $\left(V_{T E G}\right)$, these pellets are connected in a zig-zag manner, i.e., they are connected electrically in series and thermally in parallel. Fig. 2 depicts a $3 \times 3$ array of TEG pellet pairs (a total of 9 pairs) connected to a load, which is usually a converter circuitry to interface between TEG and the energy storage. When a temperature gradient is applied to this module such that the bottom side (hot side) becomes hotter than the top side (cold side), current flows through the load in the clockwise direction.

The total electrical resistance of a TEG pellet can be calculated as

$$
R_{T E G}=4 R_{\text {cont }}+R_{S L},
$$

where $R_{\text {cont }}$ is the contact resistance plus the resistance between a semiconductor pellet and its respective metal contact and $R_{S L}$ is the electrical resistance of $\mathrm{N}$ - and P-pellets. The multiplier factor of 4 on the first term accounts for four contact surfaces between pellets and contacts.

The generated voltage by TEGs is called Seebeck voltage and can be formulated as

$$
\alpha_{N} \Delta T
$$

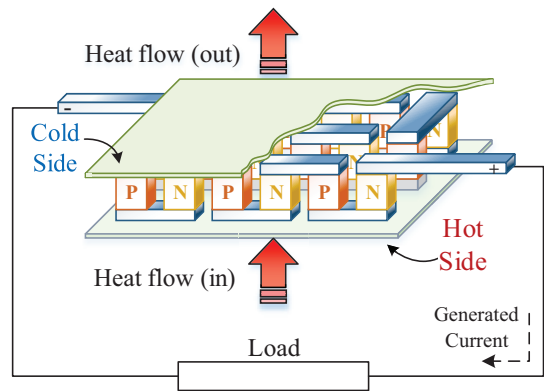

Fig. 2. A $3 \times 3$ TEG module connected to a load.

where $\alpha_{N}$ is the Seebeck coefficient of $N$ pellet pairs and $\Delta T$ is the temperature difference across them. The heat flow rate through the hot side of a TEG module $\left(\dot{q}_{h}\right)$ and its cold side $\left(\dot{q}_{c}\right)$ which result in the generation of current $I$ may be formulated as

$$
\begin{gathered}
\dot{q}_{h}=\frac{\Delta T}{\Theta_{T E G, N}}-\alpha_{N} I T_{h}-\frac{1}{2} R_{T E G, N} I^{2} \text { and } \\
\dot{q}_{c}=\frac{\Delta T}{\Theta_{T E G, N}}-\alpha_{N} I T_{c}+\frac{1}{2} R_{T E G, N} I^{2} .
\end{gathered}
$$

In these equations, $R_{T E G, N}$ and $\Theta_{T E G, N}$ are the electrical and thermal resistances of $N$ pairs of TE pellets. In this paper, the subscript $N$ denotes a parameter describing $N$ TE pairs, whereas parameters without it are related to only one pair. Accordingly, we have $\alpha_{N}=N \times \alpha, R_{T E G, N}=N \times R_{T E G}$, and $\Theta_{T E G, N}=$ $\Theta_{T E G} / N$.

Using the well-known duality between electrical and thermal phenomena [9], an electro-thermal model of $N$ TEGs can be developed as shown in Fig. 3 [8]. Note that the red part shows the thermal model, whereas the blue part designate the electrical model.

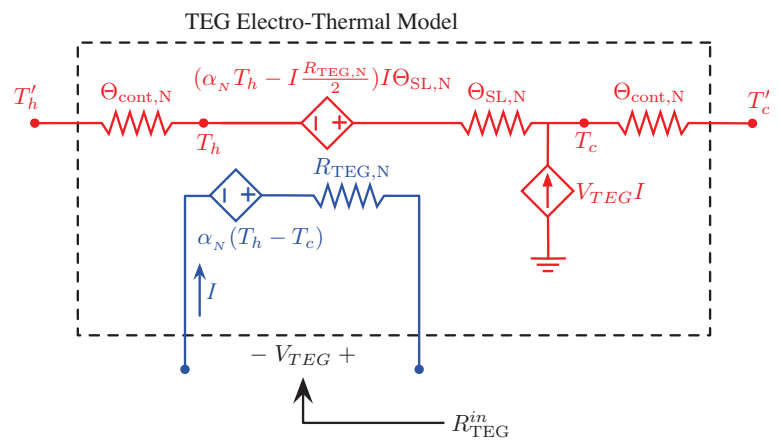

Fig. 3. Electro-thermal model of $N$ TEGs considering the contact thermal resistances. The thermal part is represented in red and the electrical part is shown in blue.

In Fig. 3, $\Theta_{S L, N}$ represents the thermal resistivity of $N$ super-lattice material pairs, whereas $\Theta_{\text {cont }, N}$ shows the thermal resistivity of metal contacts on the top or bottom of $N$ TEGs. Clearly, the following relation holds.

$$
\Theta_{T E G, N}=\Theta_{\text {cont }, N}+\Theta_{S L, N}=N\left(\Theta_{\text {cont }}+\Theta_{S L}\right)
$$

Note that each pellet is connected to two metal contacts which can be modeled by two series resistors with the value of $\Theta_{\text {cont }}$; however, pairs of pellets are thermally connected in parallel. Hence, the overall contact thermal resistance for a pair of pellets is equal to $\Theta_{\text {cont }}$. 


\section{B. Maximum Power Transfer}

As can be seen in Fig. 3, a TEG is subjected to a temperature differential through its metal contacts. The thermal and electrical contact resistances of TEGs are not negligible [4]. The contact thermal resistance $\left(\Theta_{\text {cont }, N}\right)$ causes the temperature at surfaces of a TEG module (i.e., $T_{c}^{\prime}$ and $T_{h}^{\prime}$ ) differ from the temperature on the $\mathrm{N}$ - and P-type pellets (i.e., $T_{c}$ and $T_{h}$ ). Assuming that $T_{c}^{\prime}$ and $T_{h}^{\prime}$ are set externally, values of $T_{c}$ and $T_{h}$ become dependent on the parameters of TEG shown in the thermal part. Consequently, this affects the internal resistance of the TEG. In order to determine the internal resistance, the usual procedure consists in deriving a Thevenin equivalent circuit. However, the electro-thermal model of TEGs is a non-linear circuit. The non-linearity is produced in the thermal part, where a current-controlled voltage source generates a voltage that is a quadratic function of the current $I$ in the electrical circuit. Hence, the Thevenin's theorem cannot be applied to this circuit.

Therefore, we directly find the load resistance seen by the TEG module that maximizes the power consumed in the load. Note that the maximization of conversion efficiency of TEGs is a different objective. Efficiency is maximized for large values of the load resistance [10]. However, since the TEG source energy (i.e., heat) is available for free, the conversion efficiency is not of interest and hence the objective is to maximize the power transferred to the load.

Suppose the interface circuit has the input resistance $R_{i \text { face }}^{\text {in }}$ seen from the outputs of the TEG module. $V_{T E G}$ and $I$ denote the voltage and the current, respectively, at $R_{i f a c e}^{i n}$. Clearly, the following optimization problem should be solved to find the optimal $R_{\text {iface }}^{\text {in }}\left(\right.$ called $\left.R_{\text {iface }}^{\text {in* }}\right)$.

$$
R_{\text {iface }}^{\text {in* }}=\underset{R_{\text {iface }}^{\text {in }}}{\operatorname{argmax}}\left\{V_{\text {TEG }}^{2} / R_{\text {iface }}^{\text {in }}\right\}
$$

According to the maximum power transfer theorem [10], the internal resistance of TEG module $\left(R_{T E G, N}^{i n}\right)$ should be equal to $R_{\text {iface }}^{\text {in* }}$.

Using the nodal analysis, $V_{T E G}$ as a function of $I$ can be calculated as shown below.

$$
\begin{aligned}
& V_{T E G}=\left(\frac{\alpha_{N} R_{\text {iface }}^{\text {in }} \Theta_{S L, N}}{R_{\text {iface }}^{\text {in }}+R_{T E G, N}}\right) \times \\
& \left(\frac{T_{h}^{\prime}-T_{c}^{\prime}+\alpha_{N} I \Theta_{\text {cont }, N}\left(I^{2} \Theta_{\text {cont }, N} R_{\mathrm{TEG}, \mathrm{N}}+T_{h}^{\prime}+T_{c}^{\prime}\right)}{2 \Theta_{\text {cont }, N}+\Theta_{S L, N}-\alpha_{N}^{2} I^{2} \Theta_{\text {cont }, N}^{2} \Theta_{S L, N}}\right)
\end{aligned}
$$

As expected,

$$
\lim _{\Theta_{\text {cont } \rightarrow 0}} V_{T E G}=\alpha_{N}\left(T_{h}^{\prime}-T_{c}^{\prime}\right) \frac{R_{\text {iface }}^{\text {in }}}{R_{\text {iface }}^{\text {in }}+R_{T E G, N}},
$$

indicating that as the thermal contact resistance tends to zero, $T_{h}^{\prime}$ and $T_{c}^{\prime}$ approach $T_{h}$ and $T_{c}$, respectively.

Note that we also have

$$
V_{T E G}=-I R_{i f a c e}^{\text {in }} .
$$

Solving the system of equations comprised of (8) and (10) yields an equation for $V_{T E G}$ independent of $I$. By substituting the derived $V_{T E G}$ into Eq. (7) and solving the resulting equation, we obtain three solutions of which only one is real valued. This real solution has a closed-form expression in terms of TEG parameters; however, it is lengthy and we omit it for brevity. Using the derived value for $R_{\text {iface }}^{i n *}$ (or equivalently $R_{T E G}^{i n}$ ), maximum power extraction (MPE) can be performed for TEGs.

\section{A. Simulation Setup}

\section{Simulation Results}

In this paper, we considered a thermoelectric module made by Kryotherm called TB-127-1.4-1.2 [11]. Physical parameters of this module are as follows. $N=127, \alpha=418.8 \mu \mathrm{V} / \mathrm{K}, R_{T E G}=$ $12.6 \mathrm{~m} \Omega, \Theta_{S L}=190.3 \mathrm{~K} / \mathrm{W}$, and $\Theta_{\text {cont }}=57.2 \mathrm{~K} / \mathrm{W}$.

Synopsys HSPICE was used for performing circuit simulations. After developing the method for determining $R_{T E G, N}^{i n}$, we verified the theoretical analysis by comparisons with SPICE simulations. Perfect agreement was observed.

\section{B. Simulation Results}

Sensitivity analysis: We perform sensitivity analysis on the TEG internal resistance to see how TEG parameters affect the it. The baseline value is $R_{T E G, N}=1.6 \Omega\left(R_{T E G} \times N\right)$. Accordingly, we measure how much $R_{T E G, N}$ differs from the actual internal resistance $\left(R_{T E G, N}^{i n}\right)$ by reporting $\Delta R / R_{T E G, N}^{i n}$, where $\Delta R=R_{T E G, N}^{i n}-R_{T E G, N}$. We refer to this metric as resistance mismatch ratio.

In all analyses, the TEG parameters presented earlier are fixed and $T_{c}^{\prime}$ and $T_{h}^{\prime}$ are set to $27^{\circ} \mathrm{C}$ and $57^{\circ} \mathrm{C}$, respectively. Then, one or two parameters are selected at a time and varied. Note that the figure of merit introduced in Section $\mathrm{I}$ is defined as $Z=$ $\alpha^{2} \Theta_{T E G, N} / R_{T E G, N}$. This means that increasing $\alpha$ and $\Theta_{T E G, N}$ and reducing $R_{T E G, N}$ improve the figure of merit of a TEG. Accordingly, we change these parameters and study their effect on $R_{T E G, N}^{i n}$.

First, we select $N$ and $R_{T E G}$ parameters for analysis. However, it turns out that these parameters affect both $R_{T E G, N}^{i n}$ and $R_{T E G, N}$ in the same way. In other words, $\Delta R / R_{T E G, N}^{i n}$ remains constant. Next, we change the value of $\alpha$. The result of this analysis is shown in Fig. IV-B. As can be seen, the resistance mismatch ratio increases almost linearly with incrementing $\alpha$.

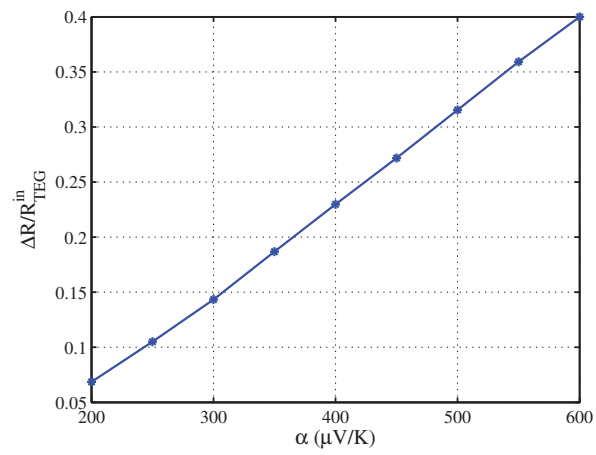

Fig. 4. Sensitivity analysis of resistance mismatch ratio on the Seebeck coefficient

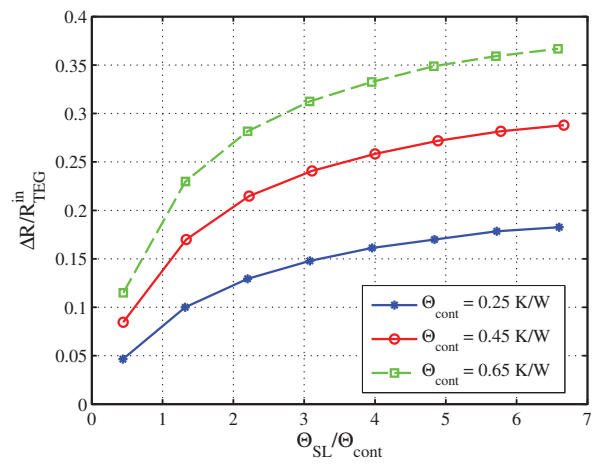

Fig. 5. Sensitivity analysis of resistance mismatch ratio on the TEG contact and supper-lattice thermal resistivity 


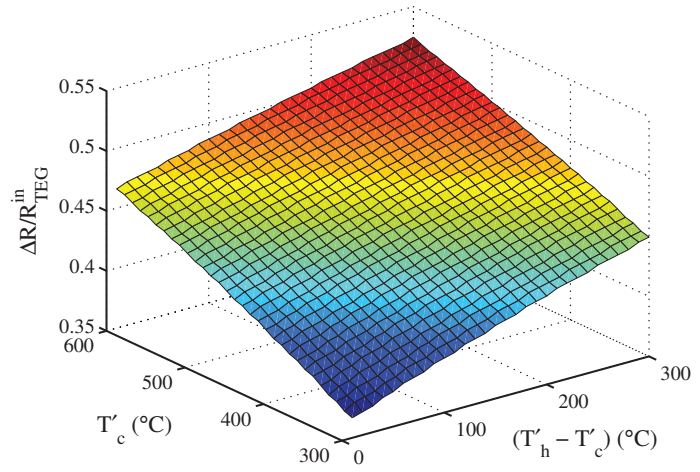

Fig. 6. Sensitivity analysis of resistance mismatch ratio on the hot and cold site temperatures of a TEG module

Next, we consider $\Theta_{S L}$ and $\Theta_{\text {cont }}$. As observed in Fig. 3 , these two parameters realize a voltage divider between $T_{c}^{\prime}$ and $T_{h}^{\prime}$. Hence, we analyze them together. Accordingly, we choose three values for $\Theta_{\text {cont }}$ and vary $\Theta_{S L}$ to investigate how changing the ratio of $\Theta_{S L} / \Theta_{\text {cont }}$ affects the resistance mismatch ratio. Fig. IV-B depicts the result. As illustrated, growth of both parameters increases the resistance mismatch ratio; however, parameter $\Theta_{\text {cont }}$ has more noticeable effect. This complies with our expectation; increasing $\Theta_{\text {cont }}$ results in larger difference between temperatures on TEG contacts $\left(T_{c}^{\prime}\right.$ and $\left.T_{h}^{\prime}\right)$ and TEG super-lattices $\left(T_{c}\right.$ and $\left.T_{h}\right)$.

Note that usually the value of $\alpha, \Theta_{S L}$, and $R_{T E G}$ are physically correlated [1]. That's why fabricating TEGs with large figure of merit is difficult. In this paper, our aim was to forecast the effect of using TEGs with larger figure of merit values on the resistance mismatch ratio.

Finally, we consider the effect of varying temperature. Fig. IV-B shows the result of changing $T_{c}^{\prime}$ and $\Delta T^{\prime}=T_{h}^{\prime}-T_{c}^{\prime}$ on the resistance mismatch ratio. As can be seen, changing $T_{c}^{\prime}$ has more significant effect on the resistance mismatch ratio compared to $\Delta T^{\prime}$. On the other hand, $\Delta T^{\prime}$ is the key parameter (along with $\alpha$ ), which determines $V_{T E G}$. With radical change in temperature, $R_{T E G, N}$ would be more than $50 \%$ smaller than the actual internal resistance $\left(R_{T E G, N}^{i n}\right)$.

A case study on ATEGs: In order to show the effect of resistance mismatch reported above, consider the case where $T_{c}^{\prime}$ and $\Delta T^{\prime}$ are selected as $700^{\circ} \mathrm{C}$ and $70^{\circ} \mathrm{C}$, respectively. These temperature values (and even larger values) are common in automotive thermoelectric generators (ATEGs), where the heat generated by the internal combustion engine is converted into electricity [1], [3], [12], [13]. Also, consider the case where $V_{O}=1.8 \mathrm{~V}$ is desirable at the output of the interface circuitry attached to the TEG module.

For a temperature difference of $\Delta T^{\prime}=70^{\circ} \mathrm{C}$, the TEG generates an open-circuit voltage of $V_{T E G}^{o c}=2.32 \mathrm{~V}$ and presents an internal resistance of $R_{T E G, N}^{i n}=3.24 \Omega$. Therefore, the maximum power that can be obtained from the TEG is $415.3 \mathrm{~mW}$ $\left(=V_{T E G}^{o c}{ }^{2} /\left(4 R_{\text {iface }}^{\text {in }}\right)\right)$, provided that $R_{\text {iface }}^{\text {in }}=3.24 \Omega$.

Now we consider how would the extracted power and the output voltage change, if we did not properly calculate the internal resistance. In this case, a Thevenin equivalent circuit would be derived, comprising a generator voltage of $3.72 \mathrm{~V}$ by using Eq. (3) and an internal resistance of $1.6 \Omega$. The voltage across the load matched to the internal resistance (i.e., 1.6 $\Omega$ ) would then be $1.86 \mathrm{~V}$. The result of this analysis would be the fact that no interface circuitry would be required, because the output voltage is already close enough to the desired voltage, i.e., $1.8 \mathrm{~V}$.
With the assumptions explained earlier, a load resistance $R_{L}=1.6 \Omega$ connected at the output of the TEG module, would cause two major problems:

1) $V_{O}$ mismatch: The voltage across the load is equal to $0.768 \mathrm{~V}$. This value differs from the target output voltage of $1.86 \mathrm{~V}$ by about 59\%. This shows that a 50\% load mismatch results in larger output deviation from what was planned.

2) Power loss: The power transferred to the load is equal to $368.2 \mathrm{~mW}$. This value is lower than the maximum power that could be extracted by more than $11 \%$.

This example illustrates the importance of proper modeling of TEGs to achieve maximum power extraction.

Note that temperature can be changed during the operation of a TEG and hence affects its internal resistance. As a result, during the design stage, the range of temperature change should be determined, and accordingly a maximum power point tracking (MPPT) system would be required to dynamically adjust the input resistance of the interface circuit. Also, note that if temperature difference changes, $V_{T E G}$ also varies. When the input voltage and the input resistance of an interface converter change simultaneously, it would be difficult to adjust with these changes. Standard MPPT techniques with proper modification can be used to address this issue (for instance, see [5].)

\section{CONCLUSION}

In this paper, it was analytically shown that the effective internal resistance of TEGs can vary from its electrical resistance by more than $50 \%$. This difference comes from ignoring the dependence of the electrical behavior of TEG on its thermal behavior. Accordingly, a systematic method for accurately determining the TEG input resistance was developed. Next, through a case study on automotive TEGs, it was shown that our method can avoid significant power loss and large voltage error across the load due to the load mismatch.

\section{ACKNOWLEDGMENT}

This project is funded in part by the Computer Systems program of the Division of Computer and Network Systems of the US NSF CISE, and the Brazilian research agencies CAPES, CNPq, and FAPERJ.

\section{REFERENCES}

[1] S. Beeby and N. White, Eds., Energy Harvesting for Autonomous Systems. Artech House, 2010.

[2] G. J. Snyder, "Small thermoelectric generators," Electrochemical Society Interface, pp. 55-57, 2008.

[3] D. M. Rowe, Ed., Thermoelectrics Handbook: Macro to Nano, 1st ed. CRC Press, Dec. 2005.

[4] I. Chowdhury et al., "On-chip cooling by superlattice-based thin-film thermoelectrics," Nature Nanotechnology, vol. 4, no. 4, pp. 235-238, 2009.

[5] Y. Ramadass and A. Chandrakasan, "A battery-less thermoelectric energy harvesting interface circuit with $35 \mathrm{mv}$ startup voltage," IEEE Journal of Solid-State Circuits, vol. 46, no. 1, pp. 333-341, Jan 2011.

[6] J. A. Federici et al., "Catalytic microcombustors with integrated thermoelectric elements for portable power production," Journal of Power Sources, vol. 161, no. 2, pp. 1469-1478, 2006.

[7] C. Lu et al., "Analysis and design of ultra low power thermoelectric energy harvesting systems," in International Symposium on Low-Power Electronics and Design, Aug 2010, pp. 183-188.

[8] S. Lineykin and S. Ben-Yaakov, "Analysis of thermoelectric coolers by a spice-compatible equivalent-circuit model," IEEE Power Electronics Letters, vol. 3, no. 2, pp. 63-66, 2005.

[9] M. Pedram and S. Nazarian, "Thermal modeling, analysis, and management in vlsi circuits: Principles and methods," Proceedings of the IEEE, vol. 94 no. 8, pp. 1487-1501, 2006

[10] D. W. Hart, Power electronics. McGraw-Hill Education, 2011.

[11] "Kryotherm." [Online]. Available: http://www.kryotherm.com

[12] L. E. Bell, "Cooling, heating, generating power, and recovering waste heat with thermoelectric systems," Science, vol. 321, no. 5895, pp. 1457-1461, 2008.

[13] "TEGs - using car exhaust to lower emissions." [Online]. Available: http://www.science20.com/news_releases/tegs_using_car_ exhaust_to_lower_emissions 